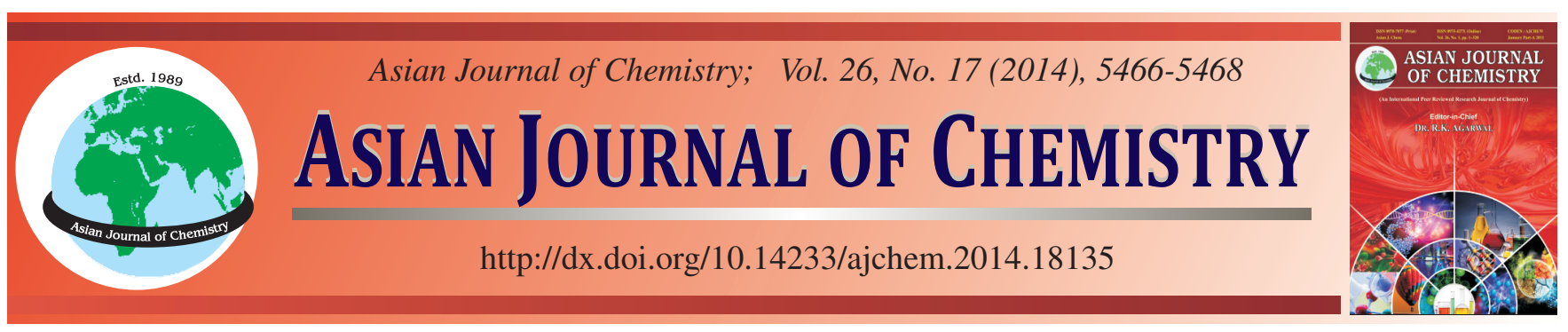

\title{
Cytocompatibility Evaluation of Hydroxyapatite Coating on Titanium Surfaces by Pulsed Electrochemical Deposition $\dagger$
}

\author{
CunPING LiU* and YANXIONG YANG
}

Modern Manufacturing Department, Yibin Vocational and Technical College, Sichuan, P.R. China

*Corresponding author: Tel./Fax: +86 831 8273013; E-mail: liucunping@126.com

\begin{abstract}
Hydroxyapatite coating was prepared on titanium substrate by pulsed electrochemical technology, in which the Ca/P ratio was 1.67 and the $\mathrm{pH}$ was adjusted by dilute $\mathrm{HNO}_{3}$ and $\mathrm{NH}_{4} \mathrm{OH}$ to 6.0. The X-ray diffraction, Fourier transform infrared spectroscopy and scanning electron microscopy were used to investigate microstructure and morphology of the materials. Then, effects of hydroxyapatite coating on the MC3T3-E1 osteoblastic cells were investigated by MTT assay method to evaluate cytocompatibility of the new scaffold. Results indicate that the prepared hydroxyapatite coating has great potential to be used as bone substitution material compared with pure titanium because of its good mechanical properties, eligible cytotoxicity and good cytocompatibility.
\end{abstract}

Keywords: Pulsed electrodeposition, Hydroxyapatite, Cytocompatibility, Titanium.

\section{INTRODUCTION}

Synthetic hydroxyapatite has been commonly used as coating material for metallic implants because of its biocompatibility and ability to form strong bonds with bones ${ }^{1,2}$. However, some mechanical properties restricted its application, such as low strength and high brittleness ${ }^{3}$. In the past decades, several coating methods have been developed to deposit hydroxyapatite on titanium surface, including plasma spraying, electrophoretic deposition, etc. ${ }^{4-9}$. Among these methods, electrochemical deposition has unique advantages due to its simple setup and capability of forming uniform coating.

In addition, the deposition process can be conducted at room temperature and the morphology of coating can be controlled easily by varying electrochemical potential and electrolyte concentration ${ }^{10}$.

In this study, the surface morphology, crystallography and chemical composition of the hydroxyapatite coatings deposited by pulsed electrodeposition were characterized using the SEM, XRD, FTIR, respectively. Then, the toxicity and biological activity of both pure titanium and hydroxyapatite coating were tested. The effect of material on osteoblast cells growth was also observed by SEM.

\section{EXPERIMENTAL}

Substrate pretreatment: Commercial pure titanium sheets (Ti, Baojint MTCO, China) were cut into specimens with dimensions of $40 \mathrm{~mm} \times 25 \mathrm{~mm} \times 1 \mathrm{~mm}$. Then the specimens were etched chemically in $2 \mathrm{M} \mathrm{H}_{2} \mathrm{O}_{2}$ and $0.1 \mathrm{M} \mathrm{HNO}_{3}$ at $310 \mathrm{~K}$ for $0.5 \mathrm{~h}$ in order to remove oxide and to increase roughness of the substrates for better adhesion. Fig. 1a showed the morphology of pure titanium after chemical treatment.

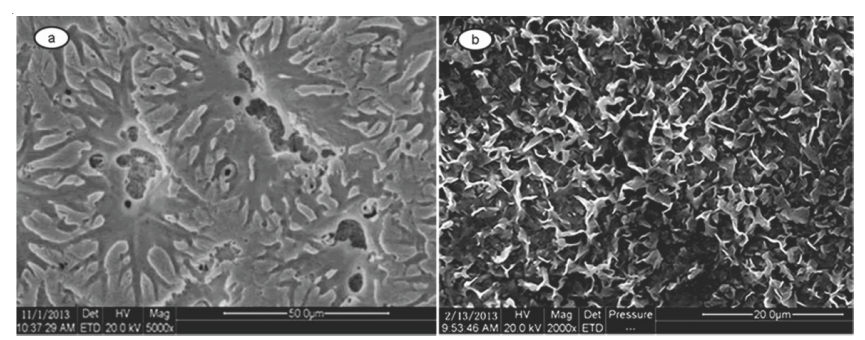

Fig. 1. (a) SEM image of pure titanium after treated with $\mathrm{H}_{2} \mathrm{O}_{2} / \mathrm{HNO}_{3}$ and (b) SEM image of electrodeposited Hap-Ti coating

Electrodeposition of hydroxyapatite: The deposition solution contained $\left(\mathrm{NH}_{4}\right)_{2} \mathrm{HPO}_{4}$ and $\mathrm{Ca}\left(\mathrm{NO}_{3}\right)_{2}$ with $\mathrm{Ca} / \mathrm{P}$ ratio 
of 1.67 in distilled water. The solution's $\mathrm{pH}$ was adjusted to 6.0 by dilute $\mathrm{HNO}_{3}$ and $\mathrm{NH}_{4} \mathrm{OH}$. The electrodeposition was carried out in a typical three electrochemical cell with constant temperature of water bath at $310 \mathrm{~K}$ for $0.5 \mathrm{~h}$. In the process, titanium substrate was used as a working electrode and acted as cathode. A Pt foil was used as the counter electrode and a standard $\mathrm{Ag} / \mathrm{AgCl}$ as the reference electrode. The electrodeposition was controlled using an Autolab Potentiostat (Aut302 FRA2) with voltaic time of $7200 \mathrm{~s}$, pulse width of $100 \mathrm{~s}$ and pulse potential of $-1.3 \mathrm{~V}$. Finally, titanium substrate was rinsed with distilled water and dried at $298 \mathrm{~K}$ in dry box.

Characterization: The X-ray powder diffraction (XRD, Rigaku 2500, Japan) patterns of all products were measured on an ART-XRAY powder X-ray diffractometer using the $\mathrm{CuK}_{\alpha}$ 1 radiation with a scanning rate of $5 \% \mathrm{~min}$. The range of scanning was $25-45^{\circ}$. The test was operated at $40 \mathrm{kV}$ and $250 \mathrm{~mA}$. The scanning electron microscope (SEM, JSM-6700F, Hitachi S4800) was operated at $20 \mathrm{kV}$. The chemical composition of the coatings was analyzed using the Fourier transform infrared spectroscopy (FTIR, Perkin Elmer 1725x).

Cell culture: Cell culture tests were performed using MC3T3-E1 osteoblastic cells. The cells were cultured in alphaMEM (Gibco, USA) supplemented with $10 \%$ fetal bovine serum (FBS, Jianghai Bio Co., China), $100 \mathrm{U} / \mathrm{mL}$ penicillin (Sigma, USA) and $100 \mu \mathrm{g} / \mathrm{mL}$ streptomycin (Sigma, USA). The cells were incubated at $310 \mathrm{~K}$ in a $5 \% \mathrm{CO}_{2}$ incubator with the medium changed every two days. When reaching the stage of confluence, the cells were harvested by trypsinization followed by the addition of fresh culture medium to create cell suspension. Cell suspension was loaded into the titanium and Hap-Ti scaffolds with a concentration of $1 \times 10^{5}$ cells/ $\mathrm{mL}$. The scaffolds were put in plastic dishes containing alphaMEM supplemented with $10 \%$ FBS and incubated at $310 \mathrm{~K}$ in a $5 \% \mathrm{CO}_{2}$ incubation. After the cells were attached on materials, $900 \mu \mathrm{L}$ fresh culture medium was added to each with the culture media changed every two days. The samples were divided into two groups: A (pure titanium groups) and B (Hap-Ti groups). After incubated for 1, 3, 7 days, respectively, the scaffolds with cells were took out and analyzed. After 20 days, the cell growth on or in the scaffolds were observed by SEM.

MTT assay and alkaline phosphatase activity tests: With the 3-(4,5-dimethylthiazol-2-yl)-2,5-diphenyltetrazolium bromide (MTT) method, The cell proliferation rate on the scaffolds was measured after incubation ${ }^{11}$. The absorbance at a wavelength of $490 \mathrm{~nm}$ was measured using a Beckman DU7400 spectrophotometer. Alkaline phosphatase (ALP) activity was measured after 1, 3 and 7 days' incubation according to Lowry with $p$-nitrophenyl phosphate as a substrate ${ }^{12}$. The scaffolds were transferred into a 24-well culture plate after being washed twice by $1.5 \mathrm{~mL}$ PBS. Then $0.05 \mathrm{M}$ 2-amino-2methylpropane and $2 \mathrm{mM} \mathrm{MgCl}_{2}(\mathrm{pH}=10.15)$ were added into each well. The mixture was incubated at $310 \mathrm{~K}$ for $1 \mathrm{~h}$ and then the reaction was terminated by adding $\mathrm{NaOH}$. The ALP activity was quantified by absorbance measurements at $410 \mathrm{~nm}$. Protein concentration was determined with a micro BCA protein assay kit, using BSA as a standard. The results of MTT and ALP activity tests were analyzed through one- way ANOVA method. When the difference was below 0.05 ( $p$ $<0.05)$, the result was considered statistically significant.

\section{RESULTS AND DISCUSSION}

Characterization of Hap-Ti coating: The XRD patterns of the Hap-Ti coatings formed onto the titanium substrate and the titanium substrate were depicted in Fig. 2a(Ti) and Fig. 2a(Hap-Ti), respectively. All reflections in Fig. 2a can be indexed readily to the standard hydroxyapatite and titanium patterns. In the same time, the XRD results indicated that hydroxyapatite coatings onto the titanium surface may be quite thin (Fig. 2a). The data of indexed calculations revealed that the material deposited on titanium substrate has a hexagonal hydroxyapatite structure with parameters of $\mathrm{Ca}_{10}\left(\mathrm{PO}_{4}\right)_{6}(\mathrm{OH})_{2}$ $(\mathrm{a}=\mathrm{b}=9.352 \AA$ and $\mathrm{c}=6.882 \AA)$ obtained from ICSD database reference pattern of hydroxyapatite (01-086-0740). The lines are coincident with those of the reference XRD pattern, which corresponds to pure hydroxyapatite crystals. As indicated in Fig. 2a, pure hydroxyapatite crystals were produced on titanium surface by the electrodeposition process when taking no account of the titanium peaks. The four hydroxyapatite characteristic diffraction peaks are corresponding to the (002), (211), (112) and (300) plane, according to JCPDS (Jiont Committee on Powder Diffraction Standards, 9-432).
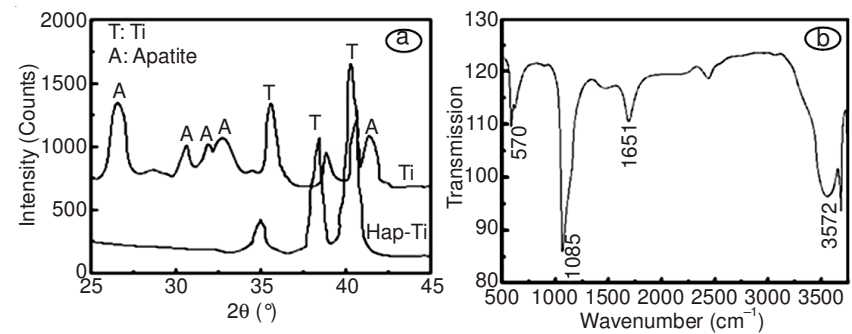

Fig. 2. (a) XRD patterns of the etched titanium substrate and Hap-Ti coating and (b) FTIR spectrum of electrodeposited coating

The FTIR spectrum of the powders scratched from the coating formed on the titanium surface were showed in Fig. 2 b. The vibration bands of 570 and $1085 \mathrm{~cm}^{-1}$ were mainly attributed to $\mathrm{PO}_{4}{ }^{3-}$. Bands corresponding to the absorption peaks of $\mathrm{OH}$ - groups appear at around 3572 and $1651 \mathrm{~cm}^{-1}$. Band at $3572 \mathrm{~cm}^{-1}$ as well as at $1651 \mathrm{~cm}^{-1}$ for absorbed $\mathrm{OH}$ - group is considered as the characteristics of hydroxyapatite. In the spectrum, the absorption peaks of $\mathrm{PO}_{4}{ }^{3-}$ and $\mathrm{OH}$ - showed strong intensities. These peaks are the signature peaks of hydroxyapatite. These were in good agreement with XRD analysis. Especially, the peak of $3572 \mathrm{~cm}^{-1}$ is considered to be the characteristic peak of hydroxyapatite ${ }^{13}$. In Fig. 1 b, The morphology of the hydroxyapatite coating can be clearly observed. The coating appears in the mixture of flake-like crystal.

MTT assay: The results of MTT assay in Fig. 3a indicate that both the Hap-Ti coating and pure titanium have a dose and time dependent effect on the cells. The amount of cells has not significant differences in the 1 day $(p>0.05)$ compared with that in the $3 \mathrm{rd}$ and 7 th day. The figure reveals that hydroxyapatite has a strong effect on growth of the cells, especially in the 7 th day $(p<0.05)$. The absorbance contrast value displays that the cytotoxicity of B group is obviously lower than that of the A group, and this situation is advantageous for growing 
satellite cells. So, biological compatibility is improved after coating hydroxyapatite on titanium.

The results of alkaline phosphatase (Fig. 3b) reveal that both the pure titanium and Hap-Ti have an effect on biological activity. The concentration of alkaline phosphatase has not significant differences between A group and B group in the 1 day $(p>0.05)$. However, Fig. $3 b$ demonstrates the subsequent changes on the alkaline phosphatase concentration in the $3 \mathrm{rd}$ and 7 th day $(p<0.05)$. Obviously, the $\mathrm{B}$ group has stronger bone inducing ability for osteoblast than the A group because of the better effect on growth and differentiation for osteoblast.
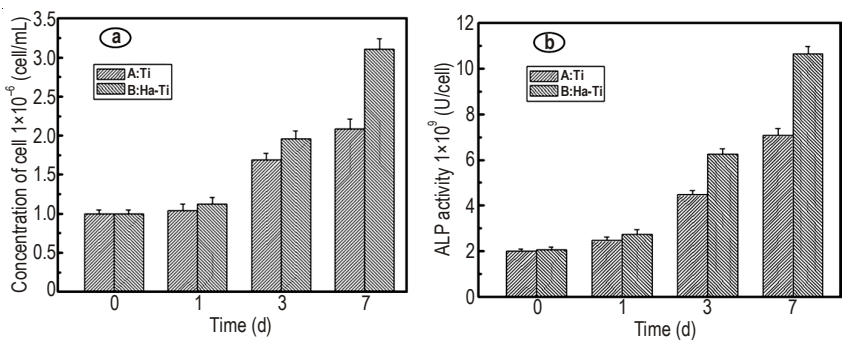

Fig. 3. (a) MTT assay for assessing adhesion of MC3T3-E1 osteoblasts and (b) ALP activity of the cells on each group material

Survey of cells on both titanium and Hap-Ti surface with SEM: The SEM images of cells on the surface of titanium and Hap-Ti after 20 days show that not only growth tendency and adherence of cultured cells are good, but also granular calcified node forms on the B group (Fig. 4a,b). These granular calcified nodes, which reflect a capacity for bone mineralization, are important indices for osteogenic differentiation. This result shows that the amount of cells on B group surface is more than that on A group surface. Close connection and sedimentation of a lot calcium salts exist between cells in Fig. 4a,b. This phenomenon proves that $\mathrm{B}$ group is more beneficial to bone formation of osteoblast and has stronger capability of bone mineralization than A group.
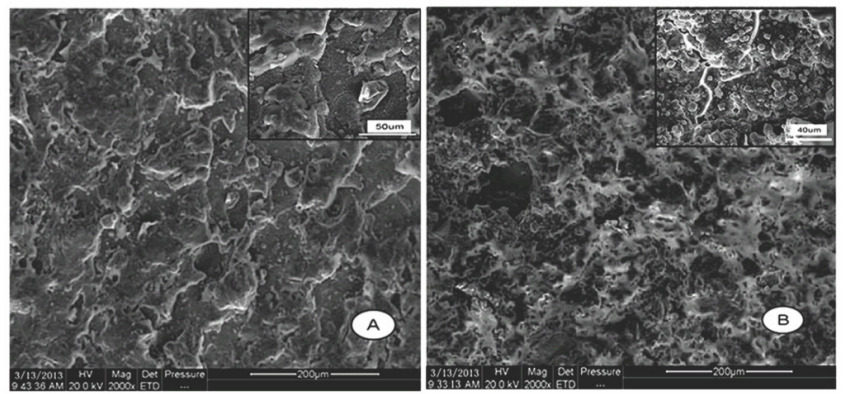

Fig. 4. (a) SEM images of cells on the surface of materials for A groups and (b) SEM images of cells on the surface of materials for B groups

\section{Conclusion}

Hydroxyapatite coatings were successfully deposited on titanium substrate by electrodeposition technique. The XRD patterns and FTIR spectrum confirm that the coatings consist of hydroxyapatite crystals. Meanwhile, the cytotoxicity of HapTi coating is obviously lower than that of pure titanium for osteoblast, and this will be advantageous for cell growth. The scaffolds of Hap-Ti coating prepared by pulsed electrochemical deposition have shown good performance for cell adhesion, growth, proliferation and osteoblast differentiation. The prepared scaffolds can be potentially employed as a biomaterial for bone reconstruction.

\section{REFERENCES}

1. R.J. Furlong and J.F. Osborn, J. Bone Joint Surg. Am., 75B, 741 (1991).

2. J. Liuyun, L. Yubao, Z. Li and L. Jianguo, J. Mater. Sci.-Mater. M, 19, 981 (2008).

3. S.K. Yen and C.M. Lin, Mater. Chem. Phys., 77, 70 (2003).

4. Nik Norziehana, Che Isa, Yusairie Mohd and Norjanah Yury, APCBEE Procedia, 3, 46 (2012).

5. H. Melero, M. Torrell, J. Fernández, J.R. Gomes and J.M. Guilemany, Wear, 305, 8 (2013).

6. X. Zhao, L. Yang, Y. Zuo and J. Xiong, Chin. J. Chem. Eng., 17, 667 (2009).

7. H. Melero, M. Torrell, J. Fernández, J.R. Gomes and J.M. Guilemany, Wear, 305, 8 (2013).

8. Sung Woon Myung, Yeong Mu Ko and Byung Hoon Kim, Appl. Surf. Sci., 287, 62 (2013).

9. A. Sarkar and S. Kannan, Ceramics Int., 40, 6453 (2014).

10. M.C. Kuo and S.K. Yen, Mater. Sci. Eng. C, 20, 153 (2002).

11. H. Wan, R. Williams, P. Doherty and D.F. Williams, J. Mater. Sci.: Mater. Med., 5, 154 (1994).

12. O.H. Lowry, N.R. Roberts, M.L. Wu, W.S. Hixon and E.J. Crawford, J. Biol. Chem., 207, 19 (1954).

13. G. Daculsi, O. Laboux, O. Malard and P. Weiss, J. Mater. Sci.-Mater. M, 14, 195 (2003). 